\title{
Control of Taenia solium taeniasis/cysticercosis: past practices and new possibilities
}

\author{
MARSHALL W. LIGHTOWLERS* \\ Department of Veterinary Science, The University of Melbourne, 250 Princes Highway, Werribee, Victoria 3030, Australia
}

(Received 7 April 2013; revised 12 May 2013; accepted 26 May 2013; first published online 15 August 2013)

S U M MAR Y

Neurocysticercosis continues to be a major health burden on humans living in many regions of the world, despite the availability of highly effective taeniacides and identification of the cause, Taenia solium, as being potentially eradicable. Several T. solium control trials have been undertaken, generally achieving limited success and none that has been fully documented has achieved what was demonstrated to be a sustainable level of disease control. Pigs act as intermediate hosts for T. solium and two new control tools have become available for application in pigs - single-dose oxfendazole treatment of porcine cysticercosis and the TSOL18 vaccine. Three potential intervention scenarios for pigs are compared for control of cysticercosis, using either oxfendazole or vaccination. A control scenario involving vaccination plus oxfendazole treatment delivered at 4 monthly intervals was predicted to achieve the best outcome, with no pigs slaughtered at 12 months of age having viable $T$. solium cysticerci. Now that new control tools are available, there are opportunities to concentrate research attention on evaluation of novel control scenarios leading to the implementation of effective and sustainable control programmes and a reduction in the global burden of neurocysticercosis.

Key words: Taenia solium, neurocysticercosis, cysticercosis, control options, vaccination, oxfendazole.

\section{INTRODUCTION}

Neurocysticercosis is one of 17 Neglected Tropical Diseases (NTD) or disease complexes recognized by the World Health Organization (2010), 8 of which are caused by helminth infections. In many ways, neurocysticercosis is one of a number of neglected NTDs because the quantum of investment in research and control activities for neurocysticercosis has been much less than has been the case for some diseases listed amongst the recognized NTDs. The reasons for neurocysticercosis being particularly neglected are many and varied. They include the lack of available data to quantify the global burden of neurocysticercosis accurately in terms such as Disability Adjusted Life Years, poor effectiveness and sustainability of methods that have been available to effect disease control, and the lack of easy-to-use animals models which facilitate laboratory-based research activities.

Neurocysticercosis is caused by infection with the larval stage of the cestode parasite Taenia solium. Humans act as both definitive and intermediate hosts for $T$. solium, although as intermediate hosts they do not play a part in the parasite's transmission. Humans become infected with the adult $T$. solium tapeworm after ingesting a mature metacestode stage (cysticercus) in uncooked, or poorly cooked, pig meat.

* Corresponding author: Department of Veterinary Science, The University of Melbourne, 250 Princes Highway, Werribee, Victoria 3030, Australia. Tel: +61 (0) 39731 2284. E-mail: marshall@unimelb.edu.au
The tapeworm grows to maturity over a period of 2-3 months (Yoshino, 1934) after which mature, infective eggs are released in the faeces. Pigs become infected with cysticerci following ingestion of items contaminated with tapeworm eggs or, as often happens, by ingesting faeces from a tapeworm carrier. Should humans ingest items contaminated with $T$. solium eggs, the parasite will also grow as a cysticercus in humans, often infecting the brain or spinal cord and causing the disease neurocysticercosis. Symptoms of neurocysticercosis vary widely depending on the number, anatomical location and patient's inflammatory response to the parasite. Many infected persons remain asymptomatic, but neurological symptoms are common with the most frequent being seizures (Garcia et al. 2003; Garcia and Del Brutto, 2005). T. solium infection is considered to be the most common preventable cause of epilepsy in the developing world (World Health Organisation, 2010).

The full life-cycle of $T$. solium is restricted to the poorest people living in many of the poorest regions of the world. The reason for this is clear from the parasite's life-cycle - in order for the parasite to be transmitted, humans must defaecate in a way that allows pigs to have access to faeces and pigs must be managed so that they gain access to human faeces, or items contaminated with human faeces. In areas where the parasite is endemic, humans defaecate in fields or other open areas, and pigs are allowed to roam freely. Transmission of neurocysticercosis from a tapeworm carrier to other humans occurs most 
commonly in the regions where the full life-cycle is perpetuated. However, due to the ease with which a tapeworm carrier can travel with their intestinal resident, transmission from tapeworm carrier to other humans may occur anywhere in the world (see, for example, Schantz et al. 1992).

Consideration of the life-cycle of $T$. solium provides a number of immediately obvious methods by which the disease might be controlled. These include sanitary disposal of faeces, personal hygiene and management of pigs such that they would be unable to access human faeces. These practices have seen $T$. solium eliminated from many regions of the world where, as recently as the 1800 s, it was prevalent (Schantz, 2002). Economic advancement in the poor areas of Central America and Africa, where the disease is currently most prevalent, would be likely to see the incidence of neurocysticercosis decline and perhaps be eliminated from these areas as well. Indeed, over the past decades, economic advancement in both Mexico and Ecuador has been credited with contributing to a marked decline in $T$. solium transmission (Flisser and Correa, 2010; Del Brutto and Del Brutto, 2012). However, for many regions of the world where neurocysticercosis remains prevalent today, the only realistic chance for there being a substantial reduction in cases in the foreseeable future would require a deliberate intervention, rather than general economic advancement.

In addition to behavioural changes affecting sanitation and the management of pigs, other tools are available which can be used towards the control of $T$. solium transmission. A variety of natural remedies have been used for treatment of tapeworm infection for centuries, but the development of safe and effective human taeniacides by Bayer, firstly niclosamide $\left(\right.$ Yomesan $^{\circledR}$ ) in 1961 and subsequently praziquantel (Biltricide ${ }^{\circledR}$ ) in the $1980 \mathrm{~s}$, represented a major advance in both the treatment of taeniasis and also the potential for control of Taenia infections. More recently, two new control measures have been developed. Gonzalez et al. (1996) discovered that a single treatment of pigs with the benzimidazole drug oxfendazole could kill all $T$. solium cysticerci in the muscles of infected pigs and a highly effective vaccine, TSOL18, has been developed (Flisser et al. 2004; Gonzalez et al. 2005) and shown to be active in field situations (Assana et al. 2010; Jayashi et al. 2012).

A number of trials have been undertaken which have sought to evaluate one control measure, or a combination of control measures, against $T$. solium. These trials provide valuable data to inform the design of future intervention strategies through which an effective and sustainable strategy may be identified and then used to reduce $T$. solium transmission. The following discussion considers the intervention practices that have been undertaken to date, with an emphasis on their effects on porcine cysticercosis, and considers new possibilities for undertaking effective and sustainable control programmes for $T$. solium in the future.

\section{PUBLIC EDUCATION}

Changes in the behaviour of people living in areas where $T$. solium is endemic can affect the parasite's transmission, either through reducing the likelihood that a person could become infected with a tapeworm (by not eating poorly cooked pork), reducing the potential for personal contamination with $T$. solium eggs (personal hygiene; washing hands after defaecation), reducing the potential for environmental contamination (by use of hygienic latrines) and by reducing the opportunity for pigs to become infected (preventing pigs from free ranging). Improvements in personal sanitation and safe disposal of faeces have benefits that extend well beyond control of T. solium, making this method for prevention of neurocysticercosis a particularly attractive option.

Several $T$. solium intervention trials have incorporated public education together with other control measures. Two investigations have studied the impact of public education alone as an intervention measure. Ngowi et al. (2008, 2009) instituted an intensive, culturally sensitive education programme in the Mbulu district of Tanzania. The study involved a randomized-control field trial involving two cohorts of smallholder pig farmers in 42 villages. Residents in 409 households from 21 villages received health education and 418 households in 21 villages acted as controls. The intervention group received a variety of both personal instruction as well as printed education material concerning aspects of hygiene, disposal of faeces and management of pigs that would be expected to reduce $T$. solium transmission. Knowledge and practices concerning $T$. solium were assessed at the beginning of the trial and at both 6 months and 10-12 months after the education programme was completed. Each participating household was provided with a sentinel pig in order to evaluate the incidence of porcine cysticercosis using an antigen ELISA test. Remarkably, despite an intensive education programme, no statistical improvement was evident in practices relating to the transmission of the disease among participants when assessed after the education programme. The only practice that was found to have changed was a reduction in the consumption of pork, but an equal reduction was evident in the villages where no intervention occurred. Ngowi et al. (2008) state that the incidence rate ratio comparing the intervention group to the control group estimated from the Bayesian model and adjusting for the baseline prevalence of porcine cysticercosis were 0.57 and $0 \cdot 51$ (95\% BCI: $0 \cdot 17,1 \cdot 4)$ using Ag-ELISA and lingual examination methods, respectively. 
Ngowi et al. (2008) found only a moderate correlation between antigen ELISA and lingual palpation used to determine the incidence of porcine cysticercosis. The antigen ELISA test is known not be specific for T. solium, cross-reacting with infection in pigs with Taenia hydatigena (Dorny et al. 2004a) and with Taenia saginata asiatica (Geerts et al.1992). The prevalence of $T$. hydatigena infection in Africa has been regarded as being low (Dorny et al. 2004b), but the evidence to support this conclusion is very limited. In addition, the potential for exposure of pigs to eggs of $T$. saginata or other Taenia species in Africa is high, and it is not known what impact such exposures could have on the antigen ELISA test. The recent discovery that transient antigen ELISApositive serology was recorded in pigs not found to be infected with either $T$. solium or $T$. hydatigena at necropsy (Devleesschauwer et al. 2013) emphasizes the need for caution in the use of antigen ELISA as a determinant of infection with, or exposure to, T. solium in pigs.

A series of studies on control of $T$. solium infections was performed in Mexico in which three control scenarios were evaluated in different rural communities, Chalcatzingo, Atotonilco and Tetelilla. In Chalcatzingo, health education was delivered to the population, in Atotonilco mass treatment with taeniacide was provided to the human population, and in Tetelilla both interventions were combined. The effects were evaluated 6 months and 42 months after the interventions. Results of the studies in Atotonilco and Tetelilla are mentioned in the following sections. In Chalcatzingo, health education led to a dramatic effect on the management of pigs. Before the intervention, $29 \%$ of the village pigs were free roaming whereas 6 and 42 months after the intervention the proportions of free roaming pigs were 6 and 4\%, respectively (Sarti et al. 1997; Flisser, 2013). Effects of the intervention on human taeniasis were significant, with a reduction of approximately $50 \%$ evident after 42 months. Effects on porcine cysticercosis were reported to have been dramatic. No cases of porcine cysticercosis were identified among 165 pigs examined by tongue palpation in pigs 6 months after the intervention, whereas 5 of 194 pigs examined before the intervention were detected with T. solium cysts (Sarti et al. 1997). Data from evaluation of pigs at 42 months after the intervention are not included in Sarti et al. (1997). However, Pawlowski et al. (2005) and Flisser and Corea (2010) cite porcine cysticercosis data at 42 months from the same trial, where it is indicated that no cysticercosis cases were detected among 334 pigs that were examined. Differences in the prevalence of porcine cysticercosis before and at 6 and 42 months after the intervention are not statistically significant (Fisher's Exact Test, $P>0 \cdot 05)$. During the trial a number of confounding circumstances occurred, including a major cholera epidemic which led to community interventions affecting certain hygiene practices, as well as poor agricultural conditions that led to the sale of 'a high proportion of the pigs' (Sarti et al. 1997). These factors complicate the interpretation of the data insofar as it indicates a positive effect of the health intervention per se.

The potential for health education as a specific intervention directed against $T$. solium is difficult to quantify from the limited data available. Certainly it cannot be confidently interpreted as having led to a major, sustained reduction in $T$. solium transmission where it has been evaluated. The effects of education on community perceptions, knowledge and, most importantly, practices have been limited also. In cases where health education has been included together with other intervention measures (discussed in more detail below), education appears to have had a limited impact. For example, Keilback et al. (1989) found that only $2 \%$ of adult participants in a T. solium control trial that included health education were able to correctly answer questions about the life-cycle of the parasite two years after the intervention. One factor limiting the widespread application of health education is the high cost of the intervention (Pawlowski et al. 2005), which also impacts the sustainability of this measure of control.

\section{TREATMENT OF TAENIASIS CARRIERS}

Sarti et al. (2000) describe a study in which mass treatment with taeniacide was provided to citizens $>4$ years of age within a total population of 3007 in the village of Atotonilco, Mexico. A number of evaluations of human and porcine cysticercosis were made 6 and 42 months after the intervention. Little change was evident in any measure other than possibly a transient decline in porcine cysticercosis (seen by serology only) after 6 months, but not 42 months.

Allan et al. (1997) provided mass taeniacide chemotherapy to the populations of 2 rural villages in Guatemala. In both villages, a significant decrease was evident in human taeniasis 10 months after the treatment $(3 \cdot 5 \%$ before, $1 \%$ after $)$, as well as in porcine cysticercosis as determined serologically (55\% before, $7 \%$ after).

Diaz Camacho et al. (1991) provided taeniacide treatment to 559 inhabitants of the village of La Curva on the Pacific Coast of Mexico. Four patients were detected with $T$. solium tapeworms at the beginning of the trial but no case of taeniasis was found 1 year later. Only one pig was found to be infected with $T$. solium at the start of the trial and none was detected as infected 1 year after the population had been treated with taeniacide. Serological assessments undertaken on the human population suggested that transmission of $T$. solium had decreased in the village following the treatment.

Following a request from Ecuadorian authorities, the WHO Parasitic Diseases Programme and Centre 
for Research and Training in Neurosciences in Quito, Ecuador, carried out a mass taeniacide treatment study in the provinces of Loja and El Oro (Cruz et al. 1989). Treatment covered $75 \cdot 8 \%$ of the total population of 13416 persons. Examination of 70 pigs at slaughter in one Canton at the start of the trial identified 8 infected pigs $(11 \cdot 4 \%)$ whereas 3 of 113 pigs $(2 \cdot 7 \%)$ were found to be infected with $T$. solium 1 year after the mass treatment. The 3 pigs found to be infected after the intervention were all $>1$ year old and were interpreted as having been infected prior to the start of the trial. The rate of taeniasis in the population at the start of the programme was assessed to be $1 \cdot 6 \%$, based on self reporting of the voiding of a tapeworm by individuals after the taeniacide treatment. None of 539 persons reassessed for taeniasis 1 year after the initial treatment were infected with tapeworms. The authors considered that the lack of re-infection after 1 year and an even prevalence of taeniasis in citizens of different age classes at the time of the first treatment (including 23 children aged 5-9 years), suggested that 'early repetition of treatment in endemic areas may be unnecessary'.

The effects of mass taeniacide treatment as a standalone intervention measure for $T$. solium are mixed, ranging from little or no effect on transmission (Sarti et al. 2000), to what seemed to have been the complete cessation of transmission (Cruz et al. 1989). Generally, at least modest impacts on $T$. solium transmission have been reported following mass treatment of the human population with a taeniacide. Certainly it could be expected that treatment of the human population to permanently remove tapeworms would impact on $T$. solium transmission as measured by the incidence of new human and porcine cases of cysticercosis. The rate at which this could be expected to occur is unclear. Direct information is not available about survival of $T$. solium eggs in the environment. Information about egg survival for other species of taeniid cestode suggest a survival time of a year or more for some eggs (Lawson and Gemmell, 1983). Persistence of infective eggs in the environment could contribute to $T$. solium transmission after treatment of the population. The relative contribution of environmental contamination with eggs, compared to a more direct transmission mode, is unclear. The possibility exists for a transient increase in transmission to occur if tapeworms that were expelled following treatment of taeniasis patients were not disposed of in a safe manner. Indeed, one instance has been reported where this may have occurred (Keilbach et al. 1989). Persistent transmission of $T$. solium after mass population treatment with a taeniacide could also be due to cases of taeniasis that did not receive treatment, or received inadequate treatment. In the Ecuadorian study, difficulties with dividing praziquantel pills led to some individuals receiving as little as $3.4 \mathrm{mg} / \mathrm{kg}$ rather than the intended $5 \mathrm{mg} / \mathrm{kg}$ of the drug. The treatment strategy also excluded children $<4$ years if age, persons with a history of epilepsy or allergies and pregnant women. Despite an intensive effort, almost one person in four was not treated. This being the case, it is somewhat surprising that the authors found no evidence of any $T$. solium transmission when assessments were undertaken 1 year after the intervention (Cruz et al. 1989).

\section{CHEMOTHERAPY OF PIGS}

Armando Gonzalez and his colleagues in Peru have discovered that a single treatment of $T$. soliuminfected pigs with $30 \mathrm{mg} / \mathrm{kg}$ of the benzimidazole drug oxfendazole killed all cysticerci in the muscle tissues (Gonzales et al. 1996; Gonzalez et al. 1997). The effectiveness of oxfendazole as a treatment for $T$. solium in pigs was confirmed independently by Sikasunge et al. (2008). Gonzalez et al. (1998) found that cysticerci killed by oxfendazole treatment were cleared from muscle tissues within 12 weeks, leaving only very small scars. However, Sikasunge et al. (2008) found that resorption of lesions created in pig muscle tissue following the oxfendazole-induced death of cysticerci may take considerably longer, even as long as 6 months in some cases. The protracted period required for these muscle lesions to disappear has been regarded as a major drawback to the use of porcine chemotherapy as a T. solium control measure (Gilman et al. 2012). Lesions arising after oxfendazole treatment may make meat from infected animals unmarketable and thereby stimulate resistance in smallholder farmers to treating their animals (Lightowlers, 2010a). Iburg et al. (2012) found that the intensity of inflammatory responses to cysticerci in the masseter muscles of an infected pig following oxfendazole treatment was affected by the intensity of infection; inflammation was reduced in pigs with heavier parasite burdens. Cysticerci in the brain of an infected pig are not affected by the drug (Gonzalez et al. 1997); it is considered, however, that brain cysticerci are unlikely to contribute significantly to T. solium transmission (Gonzalez et al. 1997; Willingham and Engels, 2006).

Pondja et al. (2012) evaluated the use of oxfendazole as a stand-alone measure for $T$. solium control in rural villages of Angónia district, north-western Mozambique. Litters of 4-month-old piglets were selected from 54 smallholder farmers, and 4 piglets from each litter randomly allocated to three groups. One pig received oxfendazole treatment at 4 months of age, one pig received oxfendazole treatment at 9 months of age and two piglets received no treatment. Serological analyses were performed by antigen ELISA and a proportion of the animals in each treatment group was necropsied at 12 months of age to identify $T$. solium infection. One of 8 animals given oxfendazole treatment at 4 months of age was found to be infected with $T$. solium at necropsy, none 
of 8 that were treated at 9 months of age was infected while 6 of 14 untreated control animals were found to be infected with $T$. solium. These differences are not statistically significant (Fishers Exact Test $P>0 \cdot 05$ ). Considering the serological data, statistically significant differences between the groups were interpreted as suggesting that the treated groups did have a lower incidence of $T$. solium infection compared to the untreated controls. As the authors acknowledge, the serological data must be considered with caution because the test used was not specific for T. solium.

The experiment undertaken by Pondja et al. (2012) was established using a randomized block design. This approach to the design of studies on T. solium control using pigs was first adopted by Huerta et al. (2001). It is an excellent approach that takes into account the focalized distribution of $T$. solium in the vicinity of a tapeworm carrier's home (Sarti et al. 1988; Garcia et al. 2003; Pawlowski et al. 2005). However, like Huerta et al. (2001), Pondja et al. (2012) did not undertake their analyses or statistical comparisons taking into account the randomized block design. Pondja et al. (2012) indicate that the 30 animals selected for necropsy were chosen randomly. Information was not provided about whether any of these 30 animals belonged to the same group that had been established within any individual household. It is possible that none of the 15 non-infected animals from the treated farms came from a property where the untreated control animals at the same property were shown to be infected. In other words, it is possible the 15 uninfected animals in the treated group may not have been exposed to $T$. solium during the trial. Had the households been selected at random, and necropsies undertaken on all 4 pigs from the selected properties, the treated and untreated groups would have been directly comparable.

It is interesting to note that the results of the studies by Pondja et al. (2012) in Mozambique found few animals that had developed $T$. solium infections between 4 months and 12 months of age (1/8), being the period between oxfendazole dosing in the first treatment group and slaughter, compared to those that had the opportunity to accumulate infections from the time of birth (the untreated group; 6/14). While these differences are not statistically significant, they highlight an interesting issue, which is the age at which pigs raised in endemic areas are first exposed to $T$. solium. There is very little data about this, and the data that are available (de Aluja et al. 1998) do not provide a clear picture (Lightowlers, 2010a).

Research is progressing towards the standardization and registration of oxfendazole as a treatment for T. solium in pigs. A consortium of scientists from Argentina, Peru and the Global Alliance for Livestock Veterinary Medicines has undertaken an assessment of oxfendazole and its metabolites, fenbendazole sulphone and fenbendazole, in relation to plasma pharmacokinetic and tissue residue profiles after oral administration to pigs at $30 \mathrm{mg} / \mathrm{kg}$. Two formulations were assessed, a commercial formulation (Synanthic ${ }^{\circledR}$ ) as well as a formulation prepared in the laboratories of San Marcos University in Lima, Peru. Performance of both products was approximately similar and, taking into consideration official maximum residue limit of the European Medicines Agency for oxfendazole (EMEA/MRL/888/03), it was calculated that the minimum withholding period for human consumption of pigs after treatment with $30 \mathrm{mg} / \mathrm{kg}$ oxfendazole was 17 days in the case of both formulations.

Alvarez et al. (2013) undertook an investigation of the use of $30 \mathrm{mg} / \mathrm{kg}$ oxfendazole (Synanthic ${ }^{\circledR}$ ) in pigs naturally infected with various nematode species (Ascaris suum, Oesophagostomum spp., Metastrongylus spp. and/or Trichuris suis). The treatment gave $100 \%$ cure when the treated animals were assessed 5 days after dosing, highlighting an additional benefit of oxfendazole as a treatment for cysticercosis in pigs.

\section{VACCINATION OF PIGS}

There have been many published studies concerning the development of vaccines against $T$. solium. Some investigators have described a degree of therapeutic effect of immunizing infected pigs with antigens (Molinari et al. 1993, 1997; de Aluja et al. 2005), but the potential for this to be adopted as a control strategy has not been further evaluated. Two prophylactic vaccines have been the subject of reports following sustained research effort and evaluation. Both are vaccines for use in pigs. Edda Sciutto and her colleagues developed a vaccine against Taenia crassiceps infection in mice (Manoutcharian et al. 1996) and the vaccine was subsequently described as inducing immunity cross-reactive to $T$. solium in pigs. A comprehensive review of the many papers that have been published concerning this vaccine can be found in Lightowlers $(2010 b)$. Three field trials have been described using the vaccine. Huerta et al. (2001) achieved a $52 \%$ reduction in the prevalence of $T$. solium in vaccinated pigs compared with control pigs. In a subsequent trial, Sciutto et al. (2007) observed 2 infected animals among 20 control pigs, whereas among vaccinated animals, 3 were infected of a total of 98 pigs. Most recently, Morales et al. (2008) found the vaccine achieved a $54 \%$ reduction in the prevalence of $T$. solium in vaccinated pigs together with an $87 \%$ reduction in the burden of parasites compared to a control group of pigs. The vaccine produced in Mexico has yet to be evaluated outside Mexico.

The other vaccine that has been developed utilizes recombinant vaccine antigens of $T$. solium that are expressed by the parasite's oncosphere life-cycle stage (Flisser et al. 2004; Gauci et al. 2012). Three different 
oncosphere antigens have been demonstrated as capable of inducing vaccine responses in pigs that protect against $T$. solium infection. One antigen has been the subject of repeated, independent testing as a vaccine, both in experimental and field trials. The TSOL18 vaccine induces $99 \cdot 5-100 \%$ protection against an experimental challenge infection with $T$. solium in pigs in experiments carried out in Mexico (2 trials), Peru, Honduras and Cameroon (Flisser et al. 2004; Gonzalez et al. 2005; Lightowlers, 2006). Two field trials have been carried out using TSOL18. The first trial was undertaken in an endemic region of Far North Cameroon (Assana et al. 2010). Three-month-old pigs were distributed as pairs to individual smallholder pig farmers in the community. All were treated with oxfendazole, not as a control strategy in itself, but to eliminate any $T$. solium infection that may have been present in the vaccine group prior to the animals being fully vaccinated (the vaccine prevents infection, it does not cure an existing infection). Control non-vaccinated pigs received the same oxfendazole treatment. At the time the animals are generally sold or slaughtered for consumption (about 12 months of age), the vaccinated and control animals were recovered and all were subjected to intensive investigation at autopsy. T. solium cysticerci were found in 20 of the 102 control animals, 15 of which had viable cyst burdens of $>400$ cysticerci. Among the 110 vaccinated animals that were assessed, no infection was observed in any animal, either viable or non-viable. The vaccine had achieved the complete elimination of $T$. solium transmission among the animals involved in the trial. The level of protection observed in this field trial was consistent with the previous independent assessments of the vaccine that had been undertaken in experimental situations. A further field trial of TSOL18 combined with a second protective antigen, TSOL16, has been completed in Peru where the vaccine was also highly effective (Jayashi et al. 2012).

INTERVENTIONS INVOLVING A COMBINATION OF METHODS

Several studies that have sought to evaluate interventions against $T$. solium have involved the combination of two or more of the methods discussed above. Keilbach et al. (1989) published the first description of an evaluation of public education for control of $T$. solium together with mass treatment of the human population with praziquantel. The study was undertaken in a rural village in the Mexican state of Guerrero. Approximately $60 \%$ of the population agreed to receive taeniacide treatment. One year after the intervention, cysticercosis prevalence was determined in pigs of less than 1 year old by tongue palpation. The prevalence was almost twice as high $(11 \%)$ after the intervention as it had been previously $(6 \cdot 6 \%)$. It is unclear whether the difference was statistically significant. When community knowledge was assessed at the end of the study, most people had not learnt the life-cycle of the parasite although they had come to understand that it was 'bad' to eat infected meat. Nevertheless, they continued to do so. The authors comment on the difficulties they had obtaining the cooperation of the community and the limitations this placed on the intervention.

Two interventions that have been discussed above referred to studies undertaken in Mexico involving either the application of health education or mass treatment of the human population with taeniacide. The same researchers also undertook a study of both interventions applied together. The study was carried out in Tetelilla in the Mexican state of Morelos. Details of the study have not been published, but some results have been provided in review publications (Sarti, 2002; Flisser, 2013). It is puzzling that the researchers found that measures of community education and changed practices relating to reducing $T$. solium transmission had improved substantially in the community receiving taeniacide treatment alone over the period of evaluation, and more so than seen in the community that received both education and taeniacide treatment (Flisser, 2013). Sarti (2002) refers to a positive outcome of the combined intervention on porcine cysticercosis, but it is not clear whether this related to the 6 or 42 month evaluation, or both.

Garcia et al. (2006) evaluated the combined application of mass treatment of the human population with taeniacide and treatment of pigs with oxfendazole in villages in the Quilcas district of the Peruvian highlands. The outcome of the study was determined using serological methods with pig sera, with samples taken before the treatments and at 4 monthly intervals for 20 months after the intervention. A significant but relatively small (about $50 \%$ ) reduction was seen in incidence of seropositivity in pigs in some or the villages where the intervention was carried out. Interpretation of the study outcomes is limited because of the use of pig serology alone to determine the effects of the intervention, as it is now known that seropositivity in enzyme immune electro transfer blot (IETB) does not correlate as highly with $T$. solium infection that can be demonstrated at necropsy (Gavidia et al. 2013) as had been believed previously (Tsang et al. 1991) reflected also in there being many times more antibody-positive pigs than pigs harbouring cysts (Gilman et al. 2012).

$T$. solium control activities have been undertaken in China using a number of intervention methods, but they have not been formally published outside the local prefectural Public Health bureaus (Pawlowski et al. 2005). Pawlowski et al. (2005) describe one such programme as follows:

“..a T. solium elimination programme was undertaken by the Anti-Epidemic Station of Wujiang County, 
Zhangye Prefecture in north-central Gansu province in 1978 when the baseline rate of human taeniasis was 1512 per 100000 for the county. A total of 312 T. solium carriers were identified after purgative treatment using the traditional medicines of areca nut and pumpkin seed extract. The porcine cysticercosis rate was $7 \cdot 7 \%$. Following bi-annual targeted treatment of carriers (including the use of praziquantel from 1983) in conjunction with emphasis on confining or restraining pigs by tying and health education propaganda in the media, the incidence of human taeniasis was officially reported to have reduced to 21 per 100000 , and the porcine rate to $0 \cdot 27 \%$, by 1988 (X. Lie, Y. Zhang, Zhanyi, Gansu, China and P. S. Craig, unpublished observations).”

It would be valuable for full details of these activities to be made available for scientific analysis. Much could be learnt by others interested in $T$. solium control through study of the methodologies and results that have been obtained in China.

Another, as yet unpublished, study on the control of $T$. solium has been underway since at least 2005 (Garcia and Del Brutto, 2005) with funding from the Bill and Melinda Gates Foundation. The programme seeks to eliminate $T$. solium transmission in the Tumbes region of northern Peru. Following an initial assessment of seven different control options, the programme has chosen to proceed with an intensive effort where control activities are now being delivered to a population of approximately 80000 inhabitants and involves three rounds of mass taeniacide chemotherapy in humans, five rounds of oxfendazole chemotherapy of pigs, and vaccination of pigs with the TSOL18 vaccine (Garcia et al. 2010; Gilman et al. 2012). The programme has been described as having been successful in reducing $T$. solium transmission, as determined by a combination of pig serology and necropsy, with no transmission detected in $95 \%$ of the target communities after the intervention (Garcia et al. 2010).

In summary, based on currently available information, the results of efforts to control $T$. solium using combinations of intervention methods have not led to outcomes that have been clearly superior to some interventions that have employed a single strategy.

\section{LIMITING FACTORS FOR T. SOLIUM CONTROL}

The full life-cycle of $T$. solium is only perpetuated in poor regions of the world where open air defaecation or other unhygienic practices concerning disposal of faeces, and free roaming pigs, facilitate parasite transmission. The concentration of disease transmission in poor regions imposes major limitations on the resources that are available for undertaking control measures and the suitability of different intervention options. The most critical issues with
T. solium control options relate to their general applicability and sustainability. Control of transmission must be achieved with a realistic and achievable level of resources, and it must be possible to sustain the reduced transmission with an acceptable level of resource inputs so that the disease does not re-establish to pre-intervention levels. Both cost and practicality are major factors. Cost has been a significant issue with intensive community education programmes (Pawlowski et al. 2005) which, for the most part, have had limited effectiveness. With the possible exceptions of the control efforts that have been undertaken in the Gansu province, China and in Peru (but yet to be described in detail), none of the specific attempts to reduce $T$. solium transmission have been undertaken for a sustained period. Hence, it has not been possible to determine whether the level of disease control described for some studies could be maintained in a sustainable manner.

The most effective strategy that has been described to date has been the use of the TSOL18 vaccine in pigs, together with oxfendazole treatment of young pigs to remove any $T$. solium infections that may have established in the animals prior to their being vaccinated (Assana et al. 2010). An important factor affecting pig vaccination as a control measure for $T$. solium is the fact that animals must be vaccinated twice in order to induce a protective immune response. However, the lack of a seasonal breeding cycle for pigs would require any intervention to be carried out either on an $a d$ hoc basis or multiple times per year, so as to ensure that all or most animals would be unable to have viable $T$. solium cysticerci at slaughter (discussed in more detail below). Other factors influencing the potential use of porcine vaccination as a control measure for $T$. solium include immunological immaturity of neonatal piglets, such that very young animals may not be able to be immunized satisfactorily, and the potential for maternally-derived antibody to interfere with immunization of some young piglets. To date, local-breed pigs in endemic areas of Cameroon and Peru have been successfully immunized at 3 months of age (Assana et al. 2010; Jayashi et al. 2012). No evidence was found to suggest that these piglets, born to sows resident in highly endemic regions, were affected by the presence of maternally-derived antibodies. Persistence for some months of maternally derived anti- $T$. solium antibodies has been described in piglets born of infected sows (Gonzalez et al. 1999), however this evidence relates to anti-cysticercus antibodies. TSOL18 is an oncosphere-specific protein (Gauci et al. 2006; Jabbar et al. 2010) and anti-TSOL18 antibodies are at low or undetectable levels in pigs harbouring mature cysticerci. For this reason, unless sows were immunized during pregnancy, maternally derived anti-TSOL18 antibodies would be unlikely to affect TSOL18 vaccine responses in piglets. 
Two limitations affect the use of oxfendazole in pigs as a control measure for T. solium. Firstly, care should be taken to ensure that treated animals are not slaughtered within the 17 day withholding period (Moreno et al. 2012) to avoid consumption of meat contamination with the drug or its metabolites. Pigs in many areas endemic for $T$. solium are commonly consumed at about 12 months of age. Since there is little or no regulation of pig management in $T$. solium-endemic regions, it may be prudent to avoid giving oxfendazole to pigs older than 9 months of age so as to minimize the likelihood that the treated animals would be consumed within the withholding period. If it were assumed that pigs given oxfendazole at 9 months of age were subsequently slaughtered at 12 months of age, those animals that had not previously been infected with $T$. solium at the time of oxfendazole treatment could be expected to be susceptible to exposure to $T$. solium eggs between the time of oxfendazole treatment and slaughter. Infections acquired in this window of susceptibility would be viable at slaughter. Knowledge is limited about the time required for $T$. solium cysticerci to develop in pigs to maturity such that the cysts would be infective for humans. Yoshino (1933) found that cysticerci were not capable of evaginating until after approximately 60 days development in the pig. In the case of $T$. ovis in sheep, which develops as a muscle-located cysticercus very similar to $T$. solium, development may be complete as early as 46 days post infection (Arundel, 1972). A figure of 6 weeks would be a conservative estimate for the minimum time that $T$. solium might require to reach an infective stage in pigs. Using a figure of 6 weeks as the minimum development time to maturity in pigs, animals in endemic regions that are slaughtered for consumption at 12 months of age have 10.5 months of life where they may be susceptible to T. solium infection, that has the potential to lead to the presence of mature, infective cysticerci at slaughter. This being the case, pigs given oxfendazole treatment at 9 months of age would be susceptible for 1.5 months to a $T$. solium infection that would have sufficient development time to reach maturity and infectivity by the time the pig was 12 months of age.

NEW OPPORTUNITIES FOR CONTROL OF T. SOLIUM

The dual discoveries of a single-dose chemotherapy for $T$. solium and the TSOL18 vaccine raise new and exciting possibilities for control of $T$. solium transmission. Lightowlers (2010a) proposed a control scheme involving two (primary and secondary) vaccination interventions in pigs per year, with the animals receiving oxfendazole at the time of the secondary vaccination. In order to ensure that all animals received the necessary two immunizations and chemotherapy before the animals reached the normal age for slaughter (12 months), the scenario entailed each pig owner's property being visited twice per year, with a 4 week interval between primary and secondary injections. This scenario required 4 visits to pig farmers during each 12 month period. The scheme was based on the highly successful smallscale control trial undertaken by Assana et al. (2010) in Cameroon. While this scenario would be likely to be effective, it would be advantageous if a control programme required less frequent intervention procedures. An improvement would be if the TSOL18 vaccine could be modified so as to provide protection after a single injection, rather than requiring two injections. The advantage this would have is much less than might first be imagined. This is because the potential for pigs to be eaten at or before 12 months of age, and the absence of a defined breeding season, appear to exclude any effective control scenario involving either vaccination or chemotherapy of pigs that would require only annual visits to pig farms.

Three possible $T$. solium control scenarios are compared for their effectiveness in Table 1. For the purpose of making the comparisons, effectiveness is defined as the possibility that pigs could contain mature infective cysticerci in their muscle tissues when slaughtered at 12 months of age. Considering an estimated time of 6 weeks for cysticerci to mature in pigs, this would see untreated pigs being susceptible to $T$. solium infection that could be mature at slaughter, for $10 \cdot 5$ of their 12 month life-span. The comparisons shown in Table 1 incorporate a limitation on the use of oxfendazole in pigs less than 1 month and more than 9 months old. In the case of the scenarios involving TSOL18 vaccination, the vaccine is provided together with oxfendazole treatment at the time of each (both primary and secondary) vaccination. The vaccination plus chemotherapy strategy is limited in the same way as chemotherapy alone; that is, animals younger than 1 month and older than 9 months are excluded from treatment. It is assumed that a pig not previously infected with $T$. solium, and not receiving two immunizations with TSOL18, would remain susceptible to $T$. solium infection. Three scenarios are considered: one involving the use of oxfendazole alone given at 6-monthly intervals to all pigs, vaccination plus oxfendazole treatment of pigs with treatments at 6-monthly intervals, and vaccination plus oxfendazole treatment of pigs with treatments at 4-monthly intervals. All scenarios take into account the aforementioned limitations concerning both chemotherapy and vaccination.

In calculating the Months of susceptibility shown in Table 1, by way of examples we will consider here how the calculations were performed for piglets either less than or $\geqslant 1$ month old at the time the first intervention occurs, in the 6-monthly vaccination + chemotherapy protocol. In relation to those less than 1 month old, the limitations mentioned above specify 
Table 1. Comparison of three different Taenia solium control scenarios on the susceptibility of pigs going to slaughter. Individual cohorts of animals receiving TSOL18 vaccination plus oxfendazole (OFZ) at 6- or 4-monthly intervals, or OFZ alone at 6-monthly intervals. Shaded cohorts represent animals that will attain slaughter age prior to the next scheduled intervention and their treatment status at the time of slaughter

\begin{tabular}{|c|c|c|c|c|c|c|}
\hline $\begin{array}{l}\text { Age of pigs } \\
\text { at first } \\
\text { intervention }\end{array}$ & Treatment & $\begin{array}{l}\text { Age of pigs } \\
\text { at second } \\
\text { intervention }\end{array}$ & $\begin{array}{l}\text { Second } \\
\text { treatment } \\
\text { or status at } \\
\text { slaughter }^{\mathrm{a}}\end{array}$ & $\begin{array}{l}\text { Age of pigs } \\
\text { at third } \\
\text { intervention }\end{array}$ & $\begin{array}{l}\text { Third } \\
\text { intervention } \\
\text { or status at }_{\text {slaughter }^{\mathrm{a}}}\end{array}$ & $\begin{array}{l}\text { Months of } \\
\text { susceptibility }\end{array}$ \\
\hline \multicolumn{7}{|c|}{ Vaccination + OFZ at 6-month intervals } \\
\hline 0 & Nil & 6 & $\mathrm{~V} 1+\mathrm{OFZ1}$ & & & $4 \cdot 5$ \\
\hline 1 & $\mathrm{~V} 1+\mathrm{OFZ1}$ & 7 & $\mathrm{~V} 2+\mathrm{OFZ} 2$ & & & 0 \\
\hline 2 & $\mathrm{~V} 1+\mathrm{OFZ1}$ & 8 & $\mathrm{~V} 2+\mathrm{OFZ} 2$ & & & 0 \\
\hline 3 & $\mathrm{~V} 1+\mathrm{OFZ1}$ & 9 & $\mathrm{~V} 2+\mathrm{OFZ} 2$ & & & 0 \\
\hline 4 & $\mathrm{~V} 1+\mathrm{OFZ1}$ & 10 & $\mathrm{~V} 1+\mathrm{OFZ1}$ & & & $6 \cdot 5$ \\
\hline 5 & $\mathrm{~V} 1+\mathrm{OFZ1}$ & 11 & $\mathrm{~V} 1+\mathrm{OFZ} 1$ & & & $5 \cdot 5$ \\
\hline 6 & $\mathrm{~V} 1+\mathrm{OFZ1}$ & 12 & $\mathrm{~V} 1+\mathrm{OFZ1}$ & & & $4 \cdot 5$ \\
\hline 7 & $\mathrm{~V} 1+\mathrm{OFZ1}$ & & & & & \\
\hline 8 & $\mathrm{~V} 1+\mathrm{OFZ1}$ & & & & & \\
\hline 9 & $\mathrm{~V} 1+\mathrm{OFZ1}$ & & & & & \\
\hline 10 & Nil & & & & & \\
\hline 11 & Nil & & & & & \\
\hline 12 & Nil & & & & & $21(33 \%)^{\mathrm{c}}$ \\
\hline
\end{tabular}

\begin{tabular}{|c|c|c|c|c|c|c|}
\hline \multicolumn{7}{|c|}{ Vaccination + OFZ at 4-month intervals } \\
\hline 0 & Nil & 4 & $\mathrm{~V} 1+\mathrm{OFZ1}$ & 8 & $\mathrm{~V} 2+\mathrm{OFZ2}$ & 0 \\
\hline 1 & $\mathrm{~V} 1+\mathrm{OFZ1}$ & 5 & $\mathrm{~V} 2+\mathrm{OFZ} 2$ & 9 & $\mathrm{~V} 3+\mathrm{OFZ} 3$ & 0 \\
\hline 2 & $\mathrm{~V} 1+\mathrm{OFZ1}$ & 6 & $\mathrm{~V} 2+\mathrm{OFZ} 2$ & 10 & $\mathrm{~V} 2+\mathrm{OFZ2}$ & 0 \\
\hline 3 & $\mathrm{~V} 1+\mathrm{OFZ1}$ & 7 & $\mathrm{~V} 2+\mathrm{OFZ} 2$ & 11 & $\mathrm{~V} 2+\mathrm{OFZ2}$ & 0 \\
\hline 4 & $\mathrm{~V} 1+\mathrm{OFZ1}$ & 8 & $\mathrm{~V} 2+\mathrm{OFZ} 2$ & 12 & $\mathrm{~V} 2+\mathrm{OFZ2}$ & 0 \\
\hline 5 & $\mathrm{~V} 1+\mathrm{OFZ1}$ & 9 & $\mathrm{~V} 2+\mathrm{OFZ2}$ & & & \\
\hline 6 & $\mathrm{~V} 1+\mathrm{OFZ1}$ & 10 & Nil & & & \\
\hline 7 & $\mathrm{~V} 1+\mathrm{OFZ1}$ & 11 & Nil & & & \\
\hline 8 & $\mathrm{~V} 1+\mathrm{OFZ1}$ & 12 & Nil & & & \\
\hline 9 & $\mathrm{~V} 1+\mathrm{OFZ1}$ & & & & & \\
\hline 10 & Nil & & & & & \\
\hline 11 & Nil & & & & & \\
\hline 12 & Nil & & & & & $0(0)^{\mathrm{c}}$ \\
\hline \multicolumn{7}{|c|}{ OFZ at 6-month intervals } \\
\hline 0 & Nil & 6 & OFZ2 & & & $4 \cdot 5$ \\
\hline 1 & OFZ1 & 7 & OFZ2 & & & $3 \cdot 5$ \\
\hline 2 & OFZ1 & 8 & OFZ2 & & & $2 \cdot 5$ \\
\hline 3 & OFZ1 & 9 & OFZ2 & & & $1 \cdot 5$ \\
\hline 4 & OFZ1 & 10 & OFZ1 & & & $6 \cdot 5$ \\
\hline 5 & OFZ1 & 11 & OFZ1 & & & $5 \cdot 5$ \\
\hline 6 & OFZ1 & 12 & OFZ1 & & & $4 \cdot 5$ \\
\hline 7 & OFZ1 & & & & & \\
\hline 8 & OFZ1 & & & & & \\
\hline 9 & OFZ1 & & & & & \\
\hline 10 & Nil & & & & & \\
\hline 11 & Nil & & & & & \\
\hline 12 & Nil & & & & & $28 \cdot 5(45 \%)^{\mathrm{c}}$ \\
\hline
\end{tabular}

\footnotetext{
a Animals highlighted in grey represent that proportion of the animal cohort that will be slaughtered at 12 months of age before the next scheduled intervention, or be too old ( $>9$ months) to receive the next scheduled intervention.

b Defined as the number of months of a 12 month life-time that pigs would have the possibility of acquiring a T. solium infection which could lead to the presence of mature viable cysts at slaughter.

c Total number of susceptibility months and, in brackets, the total proportion of the cohort's life-time where the animals would be susceptible to acquiring a viable $T$. solium infection that could be present at slaughter (see text for details). V1, V2, V3 - primary, secondary or tertiary immunization; OXF1, OFZ2, OFZ3 - first, second or third oxfendazole treatment.
}

these animals as being below the minimum age for treatment, hence they would miss the first round of treatment. While they would remain susceptible to infection, when they reached 6 months, at the time of the second intervention, the oxfendazole treatment would eliminate any infection they may have 
acquired until that time and hence, until that age, they would accumulate no months of susceptibility that could potentially lead to the presence of mature, viable cysts when slaughtered at 12 months old. When 6 months old, this cohort of pigs receive vaccination as well as chemotherapy. For the vaccine to be effective the animals require a second injection but, by the time the next intervention takes place, these animals would have reached slaughter age. Remembering the limitation that recently acquired infections require 1.5 months to reach maturity, this cohort of pigs would be susceptible to $T$. solium infection potentially leading to mature cysticerci at slaughter for the following months of life: $7-10 \cdot 5$, that is a total of 4.5 months. In relation to those piglets that were 1 month old at the time of the first treatment, they would receive treatments at the time of the first intervention but potentially remain susceptible to infection through months $2,3,4,5$ and 6 of life. However, treatment at the age of 7 months leads to the elimination of any pre-existing infection (oxfendazole) and prevention of future infections prior to slaughter age (second vaccination), hence this cohort of pigs would accumulate 0 months of susceptibility.

Both scenarios involving 6-monthly treatments leave a substantial proportion of the pigs' life-span where they are susceptible to developing mature cysts that could be present at slaughter. In the case of the use of chemotherapy alone, $45 \%$ of the life-span of cohorts of pigs receiving 6-monthly chemotherapy on a continuing basis would not be prevented from the possibility of developing mature infective cysts present at slaughter. For the vaccination plus chemotherapy option, the figure is $33 \%$. Increasing the frequency of the combined vaccination plus chemotherapy intervention to three per year (4-monthly) results in all pigs receiving two treatments before they reach slaughter age and all animals predicted to be free of mature cysts at slaughter. Increasing the frequency of oxfendazole treatment alone to 4 -monthly is less effective (28\% susceptibility) because pigs remain susceptible to $T$. solium infection after treatment. In circumstances where $T$. solium transmission was hyperendemic, chemotherapy treatment would, to some degree, be more effective than calculated here because animals previously infected with $T$. solium are relatively immune to re-infection and, if an initial infection were killed by chemotherapy, this would provide some protection lasting beyond the treatment itself (Gonzalez et al. 2001). The potential significance to immunity to re-infection following oxfendazole treatment is likely to be limited because even in hyperendemic situations, less than $50 \%$ of pigs are generally found to be infected, even at 12 months of age.

In relation to the 4 -monthly vaccination plus chemotherapy scenario that was calculated to potentially provide complete protection of pigs to $T$. solium infection, two important issues would need to be confirmed experimentally before the scenario could be recommended with confidence. The first relates to the vaccination of some piglets as young as one month of age; the second is the interval between primary and secondary vaccination being 4 months. Neither of these factors would be relevant to the scenario outlined by Lightowlers $(2010 a$ ), which has already been shown to be effective (Assana et al. 2010); however, that scenario required 4 interventions over the course of a 12 month period, rather than the 3 intervention programme proposed here.

\section{TREATMENT OF PIGS, HUMANS OR BOTH?}

Any T. solium intervention undertaken in pigs alone would not immediately impact on transmission of cysticercosis to humans. On the other hand, interventions involving the treatment of taeniasis in humans alone are unlikely to be effective unless carried out with high frequency because of the persistence of porcine cysticercosis and the establishment of new cases of taeniasis (Lightowlers, $2010 a$ ). Simultaneous treatment of tapeworm carriers together with an effective intervention programme in pigs would be ideal; however, the complexities and expense of undertaking the two types of intervention have a substantial effect on feasibility and sustainability. An intervention programme targeting both pigs and human taeniasis is being carried out currently in Peru (Garcia et al. 2010). An opportunity for another investigation of control incorporating both human and pig interventions may exist that would not require the establishment of the taeniasis intervention specifically for the purpose of $T$. solium control. In parts of Africa an on-going Schistosomiasis Control Initiative is being undertaken (Fenwick et al. 2009) some components of which involve mass treatment of the whole population with praziquantel. Theoretically, even a single instance of mass elimination of taeniasis undertaken in a region where an effective porcine intervention for $T$. solium had been in place for at least one pig generation, would result in a situation where new cases of taeniasis could not re-establish (Lightowlers, 2010a).

Although an intervention targeted solely at pigs would not immediately impact on the incidence of cysticercosis in humans, control of both human taeniasis and human cysticercosis would be achieved by a programme that involved interventions being carried out only in pigs. In a situation where an effective intervention had been in place for at least one full generation of pigs, new cases of human T. solium taeniasis could not be established. Hence, even where a pig-only intervention for $T$. solium was implemented, the incidence of human cysticercosis could be expected to decline as $T$. solium tapeworms disappeared through natural attrition. Data from investigations where mass treatment of taeniasis 
has been undertaken and the incidence of reestablishment of taeniasis has been investigated subsequently, have been interpreted as suggesting that the $T$. solium tapeworm life-span is in the order of 1-3 years (Lightowlers, 2010a). This figure is consistent with Yoshino's observations (1934) where, after infecting himself with three cysticerci, he observed the presence of proglottids in his faeces from 2 months until 2 years and 3 months, but no more.

\section{CONCLUDING REMARKS}

The identification of $T$. solium as a potentially eradicable disease by the International Task Force for Disease Eradication during their meetings through 1989-1992 (Anonymous, 1992; Schantz et al. 1993) did not lead to the establishment of substantial intervention programmes and reduction in the incidence of neurocysticercosis. It seems likely that the limited effectiveness of the intervention trials that were carried out using human chemotherapy and/or public education led to a malaise among those interested in T. solium (Pawlowski et al. 2005). The development of a single-dose chemotherapy for porcine cysticercosis and the TSOL18 vaccine herald a new era for T. solium control. There is no longer any reason for malaise; it is time for endeavours concerning $T$. solium to focus firmly on the evaluation of new control strategies and implementation of effective and sustainable interventions, leading to a reduction in the global burden of neurocysticercosis.

\section{ACKNOWLEDGEMENTS}

Dr Charles Gauci provided valuable comments on the draft manuscript.

\section{FINANCIAL SUPPORT}

The author's research programme is work was supported by the Australian National Health and Medical Research Council grants 628320 and 100354 .

\section{REFERENCES}

Allan, J. C., Velasquez-Tohom, M., Fletes, C., Torres-Alvarez, R., Lopez-Virula, G., Yurrita, P., Soto De Alfaro, H., Rivera, A. and Garcia-Noval, J. (1997). Mass chemotherapy for intestinal Taenia solium infection: effect on prevalence in humans and pigs. Transactions of the Royal Society of Tropical Medicine and Hygiene 91, 595-598.

Alvarez, L., Saumell, C., Fuse, L., Moreno, L., Ceballos, L., Domingue, G., Donadeu, M., Dungu, B. and Lanusse, C. (2013). Efficacy of a single high oxfendazole dose against gastrointestinal nematodes in naturally infected pigs. Veterinary Parasitology 194, 70-74. http://dx.doi. org/10.1016/j.vetpar.2013.01.003.

Anonymous (1992). Update: international task force for disease eradication, 1992. Morbidity and Mortality Weekly Report 41, 691, 697-691, 698. Arundel, J. H. (1972). A review of cysticercoses of sheep and cattle in Australia. Australian Veterinary fournal 48, 140-155.

Assana, E., Kyngdon, C. T., Gauci, C. G., Geerts, S., Dorny, P., De Deken, R., Anderson, G. A., Zoli, A. P. and Lightowlers, M. W. (2010). Elimination of Taenia solium transmission to pigs in a field trial of the
TSOL18 vaccine in Cameroon. International fournal for Parasitology 40, 515-519.

Cruz, M., Davis, A., Dixon, H., Pawlowski, Z. S. and Proano, J. (1989). Operational studies on the control of Taenia solium taeniasis/cysticercosis in Ecuador. Bulletin of the World Health Organization 67, 401-407.

De Aluja, A.S., Martinez, M. J. and Villalobos, A. N. (1998). Taenia solium cysticercosis in young pigs: age at first infection and histological characteristics. Veterinary Parasitology 76, 71-79.

De Aluja, A. S., Villalobos, N. M., Nava, G., Toledo, A., Martinez, J. J., Plancarte, A., Rodarte, L.F., Fragoso, G. and Sciutto, E. (2005). Therapeutic capacity of the synthetic peptide-based vaccine against Taenia solium cysticercosis in pigs. Vaccine 23, 4062-4069.

Del Brutto, O. H. and Del Brutto, V. J. (2012). Changing pattern of neurocysticercosis in an urban endemic center (Guayaquil, Ecuador) Fournal of the Neurological Sciences 315, 64-66.

Devleesschauwer, B., Aryal, A., Tharmalingam, J., Joshi, D. D., Rijal, S., Speybroeck, N., Gabriel, S., Victor, B. and Dorny, P. (2013). Complexities in using sentinel pigs to study Taenia solium transmission dynamics under field conditions. Veterinary Parasitology 193, 172-178 Diaz Camacho, S. P., Candil Ruiz, A., Suate Peraza, V., Zazueta Ramos, M. L., Felix Medina, M., Lozano, R. and Willms, K. (1991). Epidemiologic study and control of Taenia solium infections with praziquantel in a rural village of Mexico. American fournal of Tropical Medicine and Hygiene 45, 522-531.

Dorny, P., Brandt, J. and Geerts, S. (2004a). Immunodiagnostic approaches for detecting Taenia solium. Trends in Parasitology 20, 259-260. Dorny, P., Phiri, I. K., Vercruysse, J., Gabriel, S., Willingham, A. L., III, Brandt, J., Victor, B., Speybroeck, N. and Berkvens, D. (2004b). A Bayesian approach for estimating values for prevalence and diagnostic test characteristics of porcine cysticercosis. International Fournal for Parasitology 34, 569-576.

Fenwick, A., Webster, J.P., Bosque-Oliva, E., Blair, L., Fleming, F. M., Zhang, Y., Garba, A., Stothard, J. R., Gabrielli, A. F., Clements, A. C., Kabatereine, N. B., Toure, S., Dembele, R., Nyandindi, U., Mwansa, J. and Koukounari, A. (2009). The schistosomiasis control initiative (SCI): rationale, development and implementation from 2002-2008. Parasitology 136, 1719-1730.

Flisser, A. (2013). Epidemiology of neurocysticercosis in Mexico: from a public health problem to its control. In Novel Aspects on Cysticercosis and Neurocysticercosis (ed. Foyaca Sibat, H.), pp. 255-276. InTech, Rijeka.

Flisser, A. and Correa, D. (2010). Neurocysticercosis may no longer be a public health problem in Mexico. PLoS Neglected Tropical Diseases 4, e831. Flisser, A., Gauci, C. G., Zoli, A., Martinez-Ocana, J., Garza-Rodriguez, A., Dominguez-Alpizar, J.L., Maravilla, P., Rodriguez-Canul, R., Avila, G., Aguilar-Vega, L., Kyngdon, C., Geerts, S. and Lightowlers, M. W. (2004). Induction of protection against porcine cysticercosis by vaccination with recombinant oncosphere antigens. Infection and Immunity 72, 5292-5297.

Garcia, H. H. and Del Brutto, O. H. (2005). Neurocysticercosis: updated concepts about an old disease. Lancet Neurology 4, 653-661.

Garcia, H. H., Gonzalez, A. E., Evans, C. A. and Gilman, R. H. (2003). Taenia solium cysticercosis. Lancet 362, 547-556.

Garcia, H. H., Gonzalez, A. E., Gilman, R.H., Moulton, L.H., Verastegui, M., Rodriguez, S., Gavidia, C. and Tsang, V. C. (2006). Combined human and porcine mass chemotherapy for the control of T. solium. Amercian fournal of Tropical Medicine and Hygiene 74, 850-855. Garcia, H. H., Gonzalez, A. E., Rodriguez, S., Gonzalvez, G., Llanos-Zavalaga, F., Tsang, V.C. and Gilman, R. H. (2010). Epidemiología y control de la cisticercosis en el Perú [Epidemiology and control of cysticercosis in Peru]. Revista Peruana de Medicina Experimental y Salud Publica 27, 592-597.

Gauci, C. G., Jayashi, C. M., Gonzalez, A. E., Lackenby, J. and Lightowlers, M.W. (2012). Protection of pigs against Taenia solium cysticercosis by immunization with novel recombinant antigens. Vaccine $\mathbf{3 0}$ 3824-3828.

Gauci, C. G., Verastegui, M. R., Gilman, R.H. and Lightowlers, M. W. (2006). Taenia solium and Taenia ovis: stage-specific expression of the vaccine antigen genes, TSOL18, TSOL16, and homologues, in oncospheres. Experimental Parasitology 113, 272-275. Gavidia, C. M., Verastegui, M. R., Garcia, H. H., Lopez-Urbina, T., Tsang, V.C., Pan, W., Gilman, R.H., Gonzalez, A. E. and Cysticercosis Working Group in Peru (2013). Relationship between serum antibodies and Taenia solium larvae burden in pigs raised in field conditions. PLoS Neglected Tropical Diseases 7, e2192.

Geerts, S., Zorloni, A., Kumar, V., Brandt, J. R., De Deken, R. and Eom, K. S. (1992). Experimental infection of pigs with a Taenia species from Korea: parasitological and serological aspects. Parasitology Research 78, 513-515. 
Gilman, R.H., Gonzalez, A.E., Llanos-Zavalaga, F., Tsang, V.C. and Garcia, H.H. (2012). Prevention and control of Taenia solium taeniasis/cysticercosis in Peru. Pathogens and Global Health 106, 312-318. Gonzales, A. E., Garcia, H. H., Gilman, R. H., Gavidia, C. M., Tsang, V.C., Bernal, T., Falcon, N., Romero, M. and Lopez-Urbina, M. T. (1996). Effective, single-dose treatment or porcine cysticercosis with oxfendazole. American fournal of Tropical Medicine and Hygiene 54, 391-394.

Gonzalez, A. E., Falcon, N., Gavidia, C., Garcia, H. H., Tsang, V. C., Bernal, T., Romero, M. and Gilman, R. H. (1997). Treatment of porcine cysticercosis with oxfendazole: a dose-response trial. Veterinary Record $\mathbf{1 4 1}$, 420-422

Gonzalez, A. E., Falcon, N., Gavidia, C., Garcia, H. H., Tsang, V. C., Bernal, T., Romero, M. and Gilman, R. H. (1998). Time-response curve of oxfendazole in the treatment of swine cysticercosis. American fournal of Tropical Medicine and Hygiene 59, 832-836.

Gonzalez, A. E., Gauci, C. G., Barber, D., Gilman, R. H., Tsang, V. C., Garcia, H. H., Verastegui, M. and Lightowlers, M. W. (2005). Vaccination of pigs to control human neurocysticercosis. American fournal of Tropical Medicine and Hygiene 72, 837-839.

Gonzalez, A. E., Gavidia, C., Falcon, N., Bernal, T., Verastegui, M., Garcia, H. H., Gilman, R. H. and Tsang, V. C. (2001). Protection of pigs with cysticercosis from further infections after treatment with oxfendazole. American Fournal of Tropical Medicine and Hygiene 65, 15-18.

Gonzalez, A. E., Verastegui, M., Noh, J. C., Gavidia, C., Falcon, N., Bernal, T., Garcia, H.H., Tsang, V.C., Gilman, R.H. and Wilkins, P.P. (1999). Persistence of passively transferred antibodies in porcine Taenia solium cysticercosis. Cysticercosis Working Group in Peru. Veterinary Parasitology 86, 113-118.

Huerta, M., De Aluja, A. S., Fragoso, G., Toledo, A., Villalobos, N., Hernandez, M., Gevorkian, G., Acero, G., Diaz, A., Alvarez, I., Avila, R., Beltran, C., Garcia, G., Martinez, J. J., Larralde, C. and Sciutto, E. (2001). Synthetic peptide vaccine against Taenia solium pig cysticercosis: successful vaccination in a controlled field trial in rural Mexico. Vaccine 20, 262-266.

Iburg, T. M., Karlsson, M., Spang, F., Sikasunge, C. S. and Johansen, M. V. (2012). The effect of oxfendazole treatment on muscle pathology in pigs infected with Taenia solium cysticercosis. Veterinary Parasitology 190, 442-446.

Jabbar, A., Verastegui, M., Lackenby, J. A., Walduck, A. K., Gauci, C. G., Gilman, R. H. and Lightowlers, M. W. (2010). Variation in the cellular localization of host-protective oncospheral antigens in Taenia saginata and Taenia solium. Parasite Immunology 32, 684-695.

Jayashi, C. M., Kyngdon, C. T., Gauci, C. G., Gonzalez, A. E. and Lightowlers, M. W. (2012). Successful immunization of naturally reared pigs against porcine cysticercosis with a recombinant oncosphere antigen vaccine. Veterinary Parasitology 188, 261-267.

Keilbach, N. M., De Aluja, A.S. and Sarti-Gutierrez, E. (1989). A programme to control taeniasis-cysticercosis $(T$. solium $)$ : experiences in a Mexican village. Acta Leidensia 57, 181-189.

Lawson, J. R. and Gemmell, M. A. (1983). Hydatidosis and cysticercosis: the dynamics of transmission. Advances in Parasitology 22, 261-308.

Lightowlers, M. W. (2006). Cestode vaccines: origins, current status and future prospects. Parasitology 133, S27-S42.

Lightowlers, M.W. (2010a). Eradication of Taenia solium cysticercosis: a role for vaccination of pigs. International fournal for Parasitology 40, 1183-1192.

Lightowlers, M. W. (2010b). Fact or hypothesis: Taenia crassiceps as a model for Taenia solium, and the S3Pvac vaccine. Parasite Immunology 32, 701-709. Manoutcharian, K., Rosas, G., Hernandez, M., Fragoso, G., Aluja, A., Villalobos, N., Rodarte, L. F. and Sciutto, E. (1996). Cysticercosis: identification and cloning of protective recombinant antigens. Fournal of Parasitology 82, 250-254.

Molinari, J. L., Soto, R., Tato, P., Rodriguez, D., Retana, A., Sepulveda, J. and Palet, A. (1993). Immunization against porcine cysticercosis in an endemic area in Mexico: a field and laboratory study. American Fournal of Tropical Medicine and Hygiene 49, 502-512.

Molinari, J. L., Rodriguez, D., Tato, P., Soto, R., Arechavaleta, F. and Solano, S. (1997). Field trial for reducing porcine Taenia solium cysticercosis in Mexico by systematic vaccination of pigs. Veterinary Parasitology 69, 55-63.

Morales, J., Martinez, J.J., Manoutcharian, K., Hernandez, M., Fleury, A., Gevorkian, G., Acero, G., Blancas, A., Toledo, A., Cervantes, J., Maza, V., Quet, F., Bonnabau, H., De Aluja, A.S., Fragoso, G., Larralde, C. and Sciutto, E. (2008). Inexpensive anticysticercosis vaccine: S3Pvac expressed in heat inactivated M13 filamentous phage proves effective against naturally acquired Taenia solium porcine cysticercosis. Vaccine 26, 2899-2905.
Moreno, L., Lopez-Urbina, M. T., Farias, C., Domingue, G., Donadeu, M., Dungu, B., Garcia, H. H., Gomez-Puerta, L. A., Lanusse, C. and Gonzalez, A. E. (2012). A high oxfendazole dose to control porcine cysticercosis: pharmacokinetics and tissue residue profiles. Food and Chemical Toxicology 50, 3819-3825.

Ngowi, H. A., Carabin, H., Kassuku, A. A., Mlozi, M. R., Mlangwa, J. E. and Willingham, A. L., III (2008). A health-education intervention trial to reduce porcine cysticercosis in Mbulu District, Tanzania. Preventive Veterinary Medicine 85, 52-67.

Ngowi, H. A., Mlangwa, J. E.D., Mlozi, M.R.S., Tolma, E. L., Kassuku, A. A., Carabin, H. and Willingham, A. L., III (2009). Implementation and evaluation of a health-promotion strategy for control of Taenia solium infections in northern Tanzania. International fournal of Health Promotion and Education 47, 24-34.

Pawlowski, Z., Allan, J. and Sarti, E. (2005). Control of Taenia solium taeniasis/cysticercosis: from research towards implementation. International Fournal for Parasitology 35, 1221-1232.

Pondja, A., Neves, L., Mlangwa, J., Afonso, S., Fafetine, J., Willingham, A. L., III, Thamsborg, S. M. and Johansen, M. V. (2012). Use of oxfendazole to control porcine cysticercosis in a high-endemic area of Mozambique. PLoS Neglected Tropical Diseases 6, e1651.

Sarti, E. (2002). Epidemiology of Taenia solium taeniasis and cysticercosis in Mexico. In Taenia solium Cysticercosis from Basic to Clinical Science (ed. Singh, G. and Prabhakar, S.), pp. 83-90. CABI Publishing, Wallingford.

Sarti, E., Flisser, A., Schantz, P.M., Gleizer, M., Loya, M., Plancarte, A., Avila, G., Allan, J., Craig, P., Bronfman, M. and Wijeyaratne, P. (1997). Development and evaluation of a health education intervention against Taenia solium in a rural community in Mexico. American Fournal of Tropical Medicine and Hygiene 56, 127-132.

Sarti, E., Schantz, P. M., Avila, G., Ambrosio, J., Medina-Santillan, R. and Flisser, A. (2000). Mass treatment against human taeniasis for the control of cysticercosis: a population-based intervention study. Transactions of the Royal Society of Tropical Medicine and Hygiene 94, 85-89.

Sarti Gutierrez, E. J., Schantz, P. M., Lara Aguilera, R., Gomez Dandoy, H. and Flisser, A. (1988). Taenia solium taeniasis and cysticercosis in a Mexican village. Tropical Medicine andParasitology 39, 194-198.

Schantz, P. (2002). Taenia solium cysticercosis: an overview of global distribution and transmission. In Taenia solium Cysticercosis from Basic to Clinical Science (ed. Singh, G. and Prabhakar, S.), pp. 63-73. CABI Publishing, Wallingford.

Schantz, P. M., Cruz, M., Sarti, E. and Pawlowski, Z. (1993). Potential eradicability of taeniasis and cysticercosis. Bulletin of the Pan American Health Organization 27, 397-403.

Schantz, P. M., Moore, A. C., Munoz, J. L., Hartman, B. J., Schaefer, J. A., Aron, A. M., Persaud, D., Sarti, E., Wilson, M. and Flisser, A. (1992). Neurocysticercosis in an Orthodox Jewish community in New York City. New England Fournal of Medicine 327, 692-695.

Sciutto, E., Morales, J., Martinez, J. J., Toledo, A., Villalobos, M. N., Cruz-Revilla, C., Meneses, G., Hernandez, M., Diaz, A., Rodarte, L.F., Acero, G., Gevorkian, G., Manoutcharian, K., Paniagua, J., Fragoso, G., Fleury, A., Larralde, R., De Aluja, A.S. and Larralde, C. (2007). Further evaluation of the synthetic peptide vaccine S3Pvac against Taenia solium cysticercosis in pigs in an endemic town of Mexico. Parasitology 134, 129-133.

Sikasunge, C.S., Johansen, M.V., Willingham, A. L., III, Leifsson, P. S. and Phiri, I. K. (2008). Taenia solium porcine cysticercosis: viability of cysticerci and persistency of antibodies and cysticercal antigens after treatment with oxfendazole. Veterinary Parasitology 158, 57-66.

Tsang, V.C., Pilcher, J.A., Zhou, W., Boyer, A.E., Kamango Sollo, E. I., Rhoads, M. L., Murrell, K. D., Schantz, P. M. and Gilman, R.H. (1991). Efficacy of the immunoblot assay for cysticercosis in pigs and modulated expression of distinct $\operatorname{IgM} / \operatorname{IgG}$ activities to Taenia solium antigens in experimental infections. Veterinary Immunology and Immunopathology 29, 69-78.

Willingham, A. L., III and Engels, D. (2006). Control of Taenia solium cysticercosis/taeniosis. Advances in Parasitology 61, 509-566.

World Health Organisation (2010). Working to Overcome the Global Impact of Neglected Tropical Diseases, World Health Organisation. WHO/HTM/NTD/2010.1, Geneva.

Yoshino, K. (1933). Studies on the postembryonal development of Taenia solium. Pt. II. On the youngest form of Cysticercus cellulosae and on the migratory course of the oncosphaera of Taenia solium within the intermediate host. Taiwan Igakkai Zasshi (Fournal of the Medical Association of Formosa) 32, 155-158.

Yoshino, K. (1934). On the subjective symptoms caused by the parasitism of Taenia solium and its development in man. Taiwan Igakkai Zasshi (Fournal of the Medical Association of Formosa) 33, 183-194, 115. 


\section{University Library}

\section{- M M I N E R VA A gateway to Melbourne's research publications}

Minerva Access is the Institutional Repository of The University of Melbourne

Author/s:

Lightowlers, MW

Title:

Control of Taenia solium taeniasis/cysticercosis: past practices and new possibilities

Date:

2013-11-01

Citation:

Lightowlers, M. W. (2013). Control of Taenia solium taeniasis/cysticercosis: past practices and new possibilities. PARASITOLOGY, 140 (13), pp.1566-1577. https://doi.org/10.1017/ S0031182013001005.

Publication Status:

Accepted manuscript

Persistent Link:

http://hdl.handle.net/11343/41945 